\title{
Conditions de développement du Phytoplancton. III. Modé- lisation de cultures d'algues. Une approche des conditions de succession
}

\author{
A. Dauta ${ }^{1}$
}

Un modèle élaboré à partir des concepts actuels (s tockage intra-cellulaire) est basé sur des paramètres (croissance, assimilation, quotas cellulaires) déterminés expérimentalement sur huit espèces du phytoplancton d'eau douce : 1 Diatomée, I Cyanophycée, 6 Chlorophycées. Une des particularités réside dans l'expression de la chlorophylle, qui devient une variable relièe à l'état nutritionnel. Les résultats montrent qu'un tel modèle permet de rendre compte, pour une grande part, des phénomènes liés à la croissance de populations mono ou plurispécifiques, en particulier sur les points suivants : a) consommation rapide des nutriments b) consommation de luxe et stockage c) croissance différée par rapport à l'assimilation d) réduction de la croissance par l'ombre propre e) baisse de la teneur en chlorophylle dans le cas des cultures carencees f) succession des especes sans prise en compte d'effets d'auto et hétéroantagonisme.

Conditions for phytoplankton development. III Modeling of algal cultures. An approach to the conditions for succession

A model was prepared, starting with actual concepts (intracellular stocks), and was based on parameters (growth, assimilation, cellular quotas) determined experimentally for eight species of freshwater phytoplankton : 1 diatom, 1 Cyanophyceae, 6 Chlorophyceae. One of the peculiarities of the model is due to the expression of chlorophyll, which becomes a variable relying on the nutritional state. The results show that such a model accounts for a large part of the basic phenomenon of growth in single and multi-species populations; in particular the following points : a) rapid consumption of nutrients, b) luxury consumption and stockage, c) differential growth in relation to assimilation, d) reduction in growth by self-shading, e) decline in the chlorophyll level in the case of deficient cultures, f) succession of species without taking into account the effects of auto and heteroantagonism.

\section{1. - Introduction}

La composition spécifique et la succession saisonnière du phytoplancton sont généralement expliquées par une hypothèse de compétition interspécifique vis à vis des nutriments.

Chu (1942, 1943), suivi par Rhode (1948) fut un des premiers à publier une étude définissant clairement les besoins nutritionnels des algues. De telles investigations se sont brusquement généralisèes au cours des dix dernières années. Avec le recul, il apparaît que certaines mesures (Caperon 1967, Dugdale 1967. Droop 1968, Eppley et al. 1969) ont précédé les préoccupations des modélisateurs tandis que les sui-

1. Laboratoire d'Hydrobiologie (E.R.A. 702 du C.N.R.S.), Université Paul Sabatier, 118, route de Narbonne, 31062 Toulouse cedex. vantes (a partir de 1972-1975) ont souvent été entreprises pour répondre à la demande des créateurs et des utilisateurs de modèles. La plupart de ces paramètres déterminés en laboratoire ont généralement été testés en premier lieu avec des modèles simples, validés sur les résultats expérimentaux en culture. Ces vérifications permettent de tester simultanément les concepts et les hypothèses de travail (Belyanin et Kovrov 1968, Droop 1973. Tilman et Kilham 1976, Nyholm 1978, Davis et al. 1978, Brunel et al. 1982).

C'est dans cette optique que huit espèces d'algues ont été étudiées. Ces données expérimentales permettent de développer un modele qui prend en compte la température, l'éclairement et l'ombre propre, les sourceg en nutriments, les taux d'assimilation et les stocks intracellulaires en nutriments. 


\section{2. - Résultats expérimentaux}

\section{1. - Suivi de cultures}

Trois types d'expériences ont été réalisés ou utilisés :

- le suivi de cultures monospécifiques, selon des combinaisons de température, lumière et nutriments.

- l'estimation des inhibitions interspécifiques. Chaque espèce est cultivée dans les filtrats des milieux de culture des sept autres; le taux de croissance est comparé à celui de cette espèce cultivée dans du milieu neuf.

- le suivi de cultures plurispécifiques ; elles sont obtenues en ensemençant un milieu avec les cultures des huit especes en phase de croissance exponentielle. Ce mélange est réparti selon des combinaisons Température-Lumière. La culture est suivie sur une période variant entre 5 et 15 jours.

Les données relatives aux taux de croissance (Dauta 1983a) et aux quotas cellulaires en nutriments (Dauta 1983b) sont utilisées comme références.

\section{2. - Variation du quota en chlorophylle}

L'évolution du nombre de cellules et de leur contenu en chlorophylle peut être comparé dans des cultures de Coelastrum progressivement limitées en azote (fig. 1a) et en phosphore (fig. 1b).

La baisse du quota en chlorophylle se produit assez brusquement, plus vite dans le cas de l'azote que dans celui du phosphore, et seulement à partir d'un certain seuil, proche du quota minimal en azote (QoN) ou en phosphore (QoP). L'exemple donné est également valable pour les autres espèces (excepté Anabaena si limitation en N) : au moment où le taux de croissance s'annule (pour des valeurs proches de QoN ou QoP), la teneur en chlorophylle par cellule est généralement 2 à 6 fois plus basse que dans des cultures en phase exponentielle $(Q=Q$ max $)$ et jusqu'à 16 fois inférieure à la teneur normale pour Dictyosphaerium (Dauta 1983 b).

La forme de cette variation peut être approchée par une expression hyperbolique (fig. lc). Le quota en chlorophylle d'une cellule (OCh) est relié aux réserves intracellulaires d'un nutriment $\mathrm{j}$ selon la relation :

$$
\mathbf{Q C h}=\mathbf{Q C h o j}+(\mathrm{Qj}-\mathrm{Qoj})^{*} \mathrm{OChmj} /(\mathrm{Qj}+\mathrm{KChj})(1)
$$

Avec QChoj : quota minimal en chlorophylle dans le cas d'une carence du nutriment $j$, Qj et Qoj : respectivement la réserve intracellulaire à l'instant $t$, et le quota cellulaire minimal.

La constante $\mathrm{KChj}$ (Tableau I) est calculée par ajustement, respectivement pour le cas du phosphore (KChp) et pour l'azote $(\mathrm{KChN})$.

La carence en silice (Dauta 1983b) n'entraine pas de modification notable de la teneur en chlorophylle (Coombs et al. 1976, Werner 1977).

\begin{tabular}{|c|c|c|c|c|c|}
\hline Nutrinent considere & \multirow{2}{*}{$\begin{array}{c}(+N+E) \\
\text { orchl } 1\end{array}$} & \multirow{2}{*}{$\begin{array}{l}\text { ABote } \\
\text { QoChlN }\end{array}$} & \multirow{2}{*}{ Nols } & \multicolumn{2}{|c|}{ PHOSPHORE PMOA } \\
\hline Espectce & & & & ochlp & kchlp \\
\hline Scenedesmus crasgus & 430 & 30 & -75 & 70 & 0.15 \\
\hline Coelaftrum microaporum & 300 & 20 & -70 & 100 & 3 \\
\hline Dictyosphserium puleh. & 500 & 30 & -8 & 80 & 0,2 \\
\hline Sonnedesrous quadric. & 950 & 180 & -115 & 190 & 1,2 \\
\hline Fragillaria bidens & 900 & 85 & -120 & 265 & 11,5 \\
\hline Anabaena cylindrica & 120 & pas de & refuction & 50 & 1,4 \\
\hline Pediastrun boryanum & 1120 & 100 & -172 & 300 & 7 \\
\hline Manor aphidium ninutum & 114 & 20 & -28 & 43 & 16 \\
\hline
\end{tabular}

Tableau I. Paramètres relatifs aux quotas en chlorophylle.

\begin{tabular}{|c|c|c|c|c|c|c|c|c|}
\hline \multirow{2}{*}{$\begin{array}{l}\text { CRIGINE } \\
\mathrm{DU} \\
\mathrm{MILIEI}\end{array}$} & \multicolumn{8}{|c|}{ ESPECE } \\
\hline & 5. $c$ & c. In & $0, \bar{p}$ & 5.9 & 7.6 & A. C & P. B & K. m \\
\hline s. c & 0 & - & 0 & 0 & - & + & - & - \\
\hline c. m & 0 & 0 & 0 & - & 0 & + & - & - \\
\hline D. $\mathrm{p}$ & 0 & 0 & 0 & - & . & 0 & 0 & + \\
\hline 5. $q$ & - & - & 0 & 0 & + & + & + & - \\
\hline P. $b$ & 0 & - & 0 & - & 0 & + & - & - \\
\hline A. $\mathrm{c}$ & 0 & - & - & + & + & 0 & - & + \\
\hline P. b & o & - & 0 & - & - & + & 0 & 0 \\
\hline M. m & 0 & - & - & 0 & 0 & + & - & 0 \\
\hline
\end{tabular}

Tableau II. Test d'inhibition interspécifique. $O$ : pas d'inhibition, ou inhibition non significative. + ou-: inhibition ou stimulation significative (pour nos résultats, jamais supérieures à $20 \%$ du taux de croissance de référencel. S.c: Scenedesmus crassus C.m: Coelastrum microsporum D.p : Dictyosphaerium pulchellum S.q: Scenedesmus quadricauda F.b: Fragillaria bidens A.c: Amabaena cylindrica P.b : Pediastrum boryanum M.m : Monoraphi. dium minutum 

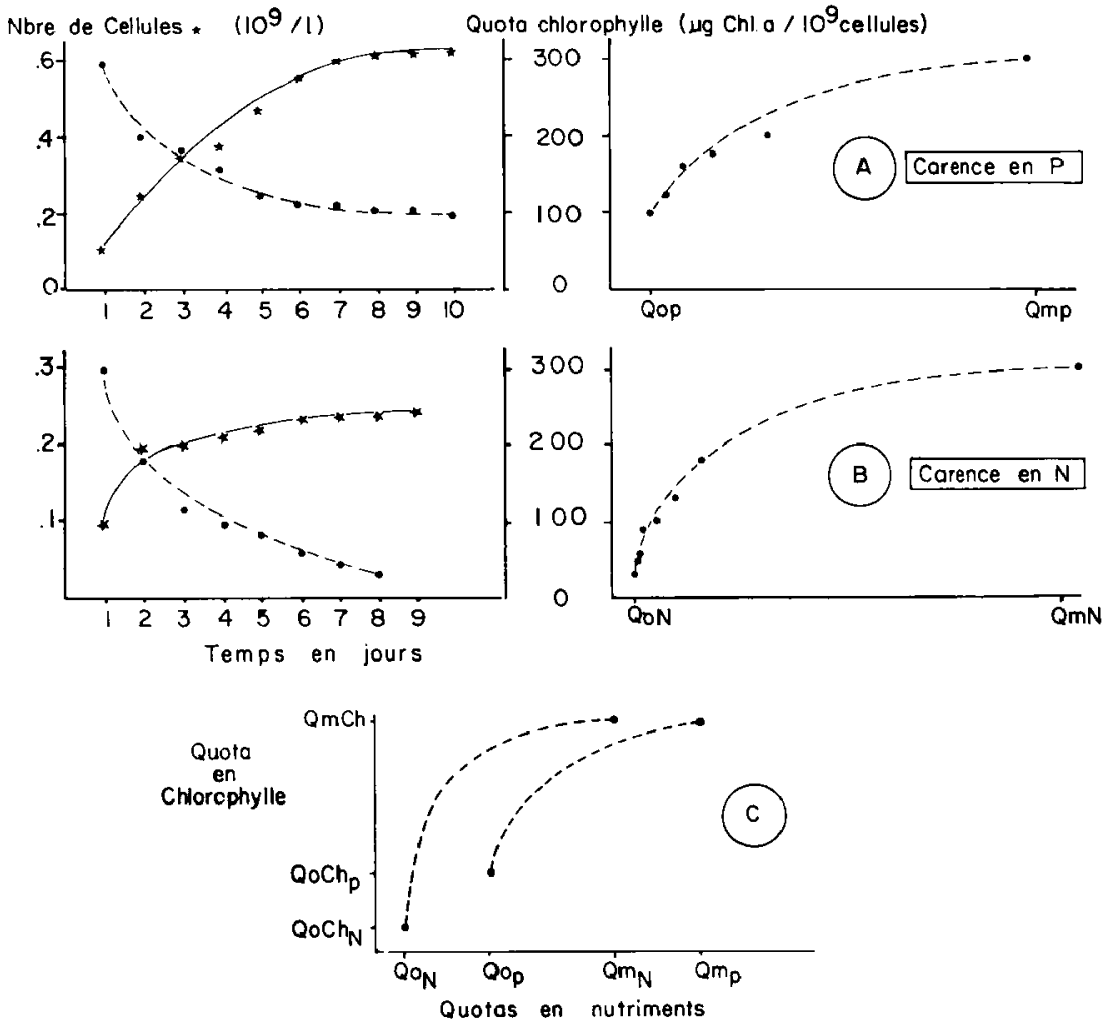

Fig. 1. Variation du quota cellulaire en chlorophylle selon la carence. (cas de Coelastrum microsporum). a) Carence en phosphore b) carence en azote c) relation entre réserves cellulaires en nutriments et le quota en chlorophylle : modèle proposé.

\section{3. - Tests d'inhtbition interspécifique}

Le Tableau II reporte les résultats des tests effectués : différence de croissance entre espèce poussant seule, et dans le milieu de culture d'une autre algue.

Certaines espèces paraissent peu sensibles: il s'agit de Scenadesmus crassus et de Dictyosphae. rium. Anabena semble insensible aux autres espè- ces. En aucun cas une baisse ou une augmentation notable du taux de croissance n'est significative.

Ces tests auraient mérité d'être réalisés dans des conditions nutritionnelles variées ; effectués ici dans le cas de cultures exponentielles, ils n'ont pas mis en évidence d'effets stimulants ou inhibiteurs importants et suffisamment nets pour être quantifiés et pris en compte par un modele. 


\section{3. - Structure du modèle}

\section{1. - Ecriture}

La formulation adoptée ici combine assimilation, quotas et croissance. La figure $\mathbf{2}$ donne le schéma général du modèle.

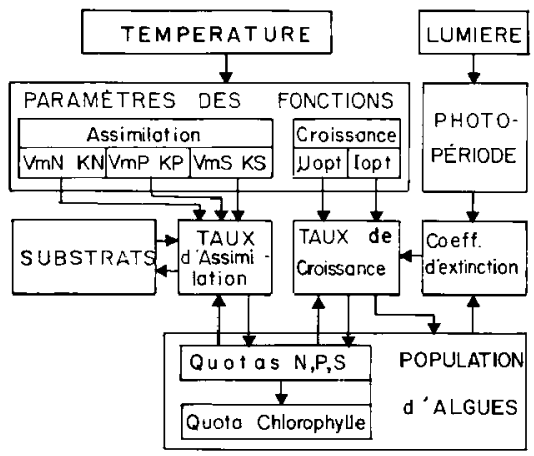

Fig. 2. Schéma du modèle.

\subsection{1-Croissance.}

L'évolution de la population est décrite en utilisant le taux de croissance, sous la forme d'un taux maximal affecté de facteurs de réduction pour des conditions non optimales de température $(\mathbf{T})$. lumière (I) et nutriments (N, P, Si) :

$$
\mu=\underset{\operatorname{RedSi}(2)}{\mu} \operatorname{RedI}^{*} \operatorname{RedT} * \operatorname{RedN} * \operatorname{RedP} \text { * }
$$

Dans ce modèle, la lumière intervient directement dans la formulation: le métabolisme du carbone (photosynthèse, respiration) est implicitement pris en compte.

Lumière et température

L'équation de Steele (1962) est utilisée pour exprimer le taux optimal de croissance lié aux conditions lumineuses :

$$
\begin{aligned}
& \mu(I, T)=\mu m(T) * a *{ }^{*} \exp \left(1-a *{ }^{*} I\right), \\
& \text { et } a=1 / \operatorname{Iopt}(T)(3)
\end{aligned}
$$

$\mu \mathrm{m}(\mathrm{T})$ et lopt $(\mathrm{T})$ sont décrits en fonction de la température selon un polynome ajusté (Dauta 1983a).

L'atténuation de la lumière incidente (Ii) par l'ombre propre est calculée par une Loi de Beer. Lambert :

$\mathrm{I}=\mathrm{Ii}^{*} \exp (\mathrm{K}$ * Dopt $)$ où $\mathrm{K}$ est une constante liée à la géométrie du système de culture utilisé (Livansky 1979) et Dopt la somme des densités optiques relatives à chaque espèce.

Réduction du taux de croissance par l'état nutritionnel

${ }_{\mu}={ }_{\mu} \mathrm{m}(\mathrm{I}, \mathrm{T}){ }^{*}(\mathrm{Qj}$-Qoj)/(Qmj-Qoj) (4) où Qj, Qoj et Qmj sont respectivement, pour un nutriment $\mathrm{J}$, le quota à l'instant $\mathbf{t}$, le quota minimal et le quota maximal.

Une loi multiplicative des carences est utilisée, soit :

Rd $=$ RedN * RedP * RedSi (Droop 1973, Lehman et al. 1975).

\subsection{2. - Assimilation et stockage intracellulaire des nutriments.}

La vitesse d'assimilation des nutriments est contrôlée à la fois par la concentration de l'élément $J$ (Si) dans le milieu de culture (Loi de MichaelisMenten) et par le stock intracellulaire $Q \mathrm{j}$, soit :

$$
\begin{aligned}
& \mathrm{Vj}=V \mathrm{mj} \text { * }[\mathrm{Sj} /(\mathbf{S j}+\mathbf{K j})] *[(\mathbf{Q m j}-\mathrm{Qj}) /(\mathrm{Qmj} \text {. } \\
& \mathrm{Qoj})](5) \\
& \mathrm{dSj}=-\mathrm{Vj}^{*} \mathrm{~N} \text { et } \mathrm{dQj}=\mathrm{Vj}-\mu{ }^{*} \mathrm{Qj}(6)
\end{aligned}
$$

La vitesse maximale d'assimilation (Vmj) et la constante de demi-saturation $(\mathrm{Kj})$ sont décrites pour $\mathrm{NNO}_{3}, \mathrm{PPO}_{4}$ et $\mathrm{SiO}_{2}$, en fonction de la température, par un polynome ajusté (Dauta 1983b).

Pour l'azote, le cas d'Anabaena est traité séparément par le modèle. La fixation de l'azote élémentaire $\mathrm{N}_{2}$ est décrite de façon implicite, et sans doute justement criticable : si la concentration en nitrate s'abaisse au dessous d'une valeur seuil fixé à 0,2 $\mu \mathrm{g}$-at. I-1, le modèle considère que Anabaena utilise $\mathrm{N}_{2}$ comme substrat, et que son quota cellulaire en azote tend vers le stock maximal. Cette extrapolation est basée, d'une part sur le fait que de très faibles concentrations en azote nitrique inhibent l'induction d'hétérocystes (Horne et al. 1979) et bloquent la formation de nitrogénase (Ogawa et Carr 1969), d'autre part sur le fait que la croissance est 
peu affectée par la limitation en azote nitrique ou ammoniacal (Cobb et Myers 1964).

\subsection{3. - Teneurs en chlorophylle.}

De notables variations dans la composition des cellules sont enregistrées lors de carences en éléments nutritifs (Rhode 1948, Holmes 1966), produisant des effets plus marqués pour l'azote (Gramms et Boyle 1972, Healey et Hendzel 1975). L'analyse de nos résultats montre que la teneur en chlorophylle $(\mathrm{QCh})$ peut être relié aux réserves en azote (QN) ou phosphore (QP) selon une loi hyperbolique, soit :

$\mathrm{QCh}=$ valeur Minimale de l'équation (1) appliquée à $\mathbf{N}$ et $\mathbf{P}$ (7).

\section{2. - Variables}

Les variables des forçage introduites par le calcul sont l'intensité lumineuse incidente (Ii), le coef. ficient d'extinction lié au système de culture, et la température.
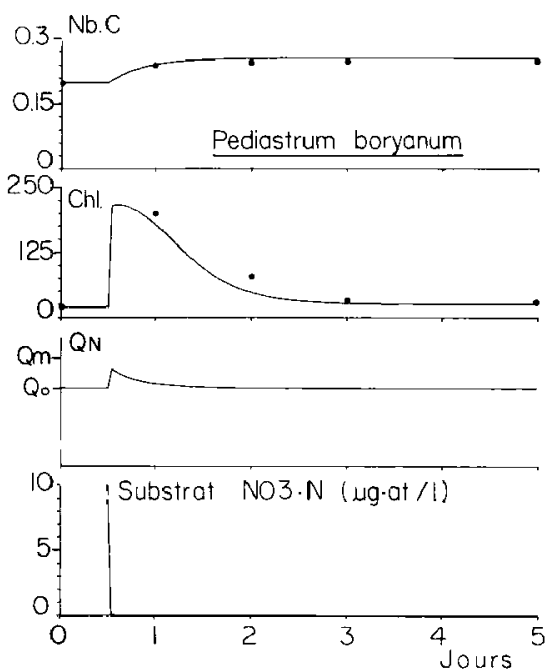

Les variations d'état caractéristiques sont:

- la population $(\mathrm{N})$, en nombre de cellules. 1-1

- les quotas cellulaires en azote (Qn), en phosphore (Qp) et pour Fragillaria, en silice (QSi)

- les quotas en chlorophylle (OCh)

- les substrats en azote (SN), phosphore (SP) et silice (SSi) après initialisation.

Les calculs s'effectuent avec un pas de temps très cours ( 0,1 à 1 heure), et les dérivés sont assimilées à des taux.

\section{4. - Modélisation}

Si la bonne restitution des phénomènes a pu être démontrée pour des valeurs instantanées de crois. sance (Dauta 1983a) ou d'assimilation (Dauta 1983b), il est indispensable de tester la validité du modèle proposé dans le cas de prédictions à long terme. De cette façon, les divers cas étudiés sont englobés : carence progressive, ajout de nutriments, ombre propre. L'évolution prédite de plusieurs populations
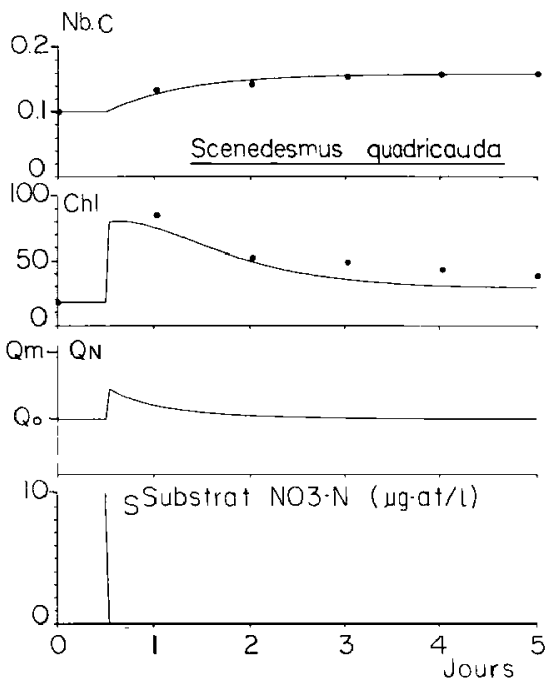

Fig. 3. Cultures monospécifiques. Carence et ajout d'azote. Cas de Pédiastrum boryanum et de Scenedesmus quadricauda. - : points mesurés - - : simulation.

Nb.c : nombre de cellules en $10^{9} \quad 1^{* 1}$. Chl : chlorophylle en $\mu \mathrm{g} .1^{* 1}$. 

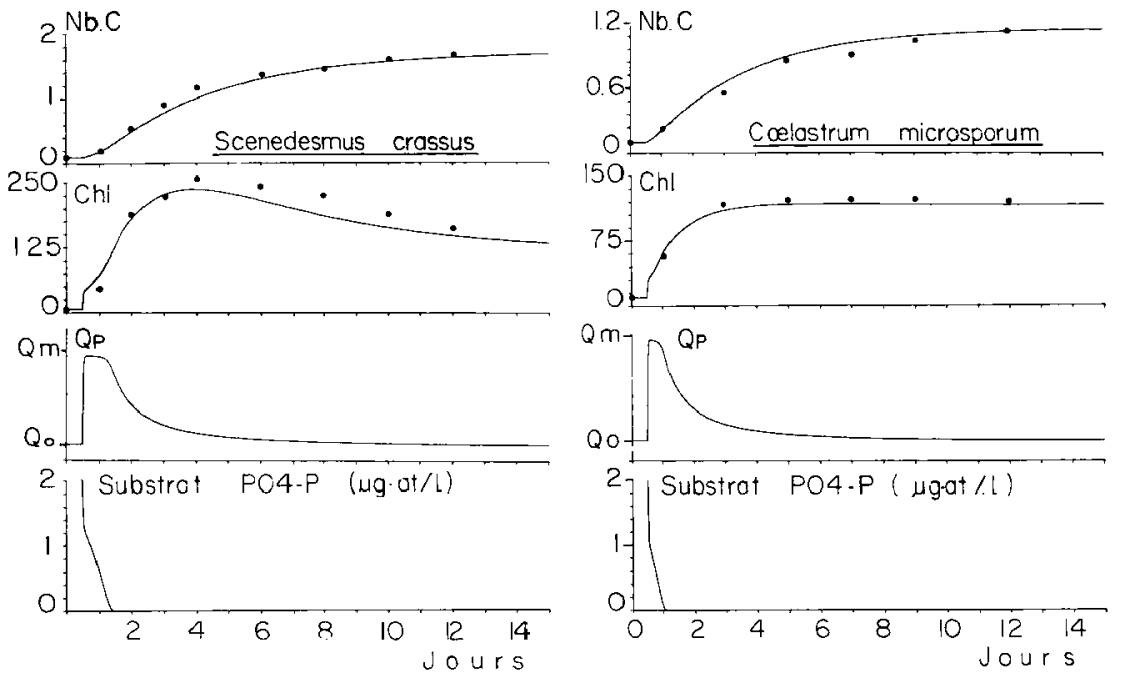

Fig. 4. Cultures monospécifiques. Carence et ajout de phosphore. Cas de Scenedesmus crassus et de Coelastrum microsporum.

- : points mesurés - : simulation.

Nb.c : nombre de cellules en $10^{9}, 1^{1 /}$. Chl. chlorophylle en $\mu \mathrm{g} .1^{1}$.

est alors comparée aux résultats expérimentaux recueillis sur des cultures en batch, mono ou plurispécifiques.

\section{1. - Cultures monospécifiques limitées en nutriments}

Les résultats de la modélisation sont comparés aux observations expérimentales (fig. 3 et 4).

Les points correspondants aux quotas extrêmes en chlorophylle sont bien restitués : en réalité, il ne peut en être autrement puisque ce sont les valeurs expérimentales introduites dans l'équation (1). Seules les valeurs intermédiaires sont intéressantes : le modèle restitue assez bien la baisse de chlorophylle d'une culture carencée vieillissante et un reverdissement si l'on rajoute le nutriment absent. Si la pré- diction de ce dernier phénomène est relativement bonne quant à la valeur atteinte, la rapidité de reconstitution du stock de chlorophylle par cellule est net tement surévaluée (en 2 heures dans le cas du modèle, contre 12 à 24 heures dans la réalité).

L'évolution de la population après l'ajout est bien simulée et le découplage entre l'assimilation et la croissance est bien restitué.

\section{2. - Cultures plurispécifiques}

\subsection{1. - Conditions non limitantes en nutriments}

Pour des conditions non limitantes en nutriments, il est possible de calculer et de comparer les taux de croissance instantanés des espèces, et ce pour la plage de température $7-35^{\circ} \mathrm{C}$ et une gamme de lumière de 0 à $750{ }_{\mu}$ E.m-2.s-l. Ainsi pour chaque 

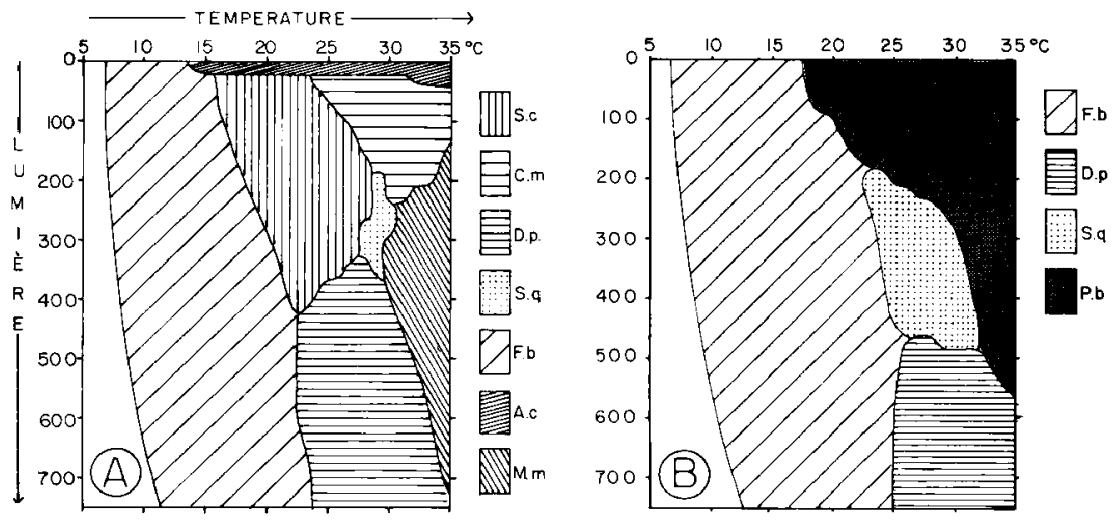

Fig. 5. Taux de croissance, combinaisons lumière - température. A) Taux de croissance instantané S.c : Scenedesmus crassus D.p : Dictyosphaérium pulchellum F.b : Fragillaria bidens P.b : Pédiastrum boryanum. B) Taux d'accroissement de biomasse C.m : Coelastrum microsporum S.q : Scenedesmus quadricauda A.c : Anabaena cylindrica M.m : Monoraphidium minutum

combinaison Température-Intensité lumineuse, il est possible de déterminer l'espèce qui possède le taux de croissance le plus élevé (fig. 5a).

De 7 à $15^{\circ} \mathrm{C}$, quelque soit la lumière, le taux de croissance de Fragillaria est supérieur à toutes les autres espèces. Le domaine des faibles lumières est favorable à Anabaena. A partir de $15^{\circ} \mathrm{C}$, la Diatomée cède peu à peu et d'abord aux faibles lumières. Au delà de $22^{\circ} \mathrm{C}$, cinq des six espèces de Chlorophycées étudiées arrivent à se distinguer : chacune devenant la plus compétitive dans une plage de Température-Lumière parfaitement délimitée. Seul Pediastrum boryanum n'apparait pas, son taux de croissance maximal $(=1,25)$ le plaçant toujours en retrait. Cependant, l'expression de cette croissance peut être pondérée par le biovolume (B) respectif de chaque espece $\left(\mu^{*} B\right)$, et exprime alors un accroissement de biomasse ; ce mode d'expression du taux de croissance avantage alors les espèces ayant des cellules de forte taille (fig. 5b) telle Fragillaria ou Pediastrum au dépens d'espèces ayant un fort taux de croissance mais de petites cellules (Monoraphidium).

Les figures $6 \mathrm{a}$ et $6 \mathrm{~b}$ retracent la dynamique de populations plurispécifiques non limitées en nutriments et pour quatre températures.
On peut noter qu'il existe des écarts, de faible amplitude, au niveau des paramètres globaux (Densité de cellules, chlorophylle) lorsque les cultures vieillissent : la population mesurée est toujours un peu inférieure à celle prédite. La composition qualitative de la population n'est pas toujours très précisément simulée, mais les grandes tendances sont respectées : si Fragillaria est l'espèce la plus efficace aux faibles températures $\left(15^{\circ} \mathrm{C}\right)$, elle est fortement concurrencée au dessus de $20^{\circ} \mathrm{C}$ lorsque la lumière disponible baisse.

Le rôle de la lumière de la lumière paraît effectivement prépondérant. Il est bien illustré par l'évolution des taux de croissance dans le temps, quelle que soit la température, des conditions initiales de fort éclairement (intensité lumineuse élevée et supérieure ou égale à $500{ }_{\mu}$ E.m-2.s-l) favorisent la croissance de l'espèce la moins sensible. C'est seulement lorsque celle-ci, par son développement, aura atténué la lumière, que les autres espèces vont commencer à croître, l'élévation du taux de croissance d'Anabaena se produisant en dernier lieu.

L'intensité lumineuse ou bien la concentration de cellules au départ revêtent alors une grande importance. De même, l'utilisation d'un système de culture diffèrent (variation du coefficient d'extinction 

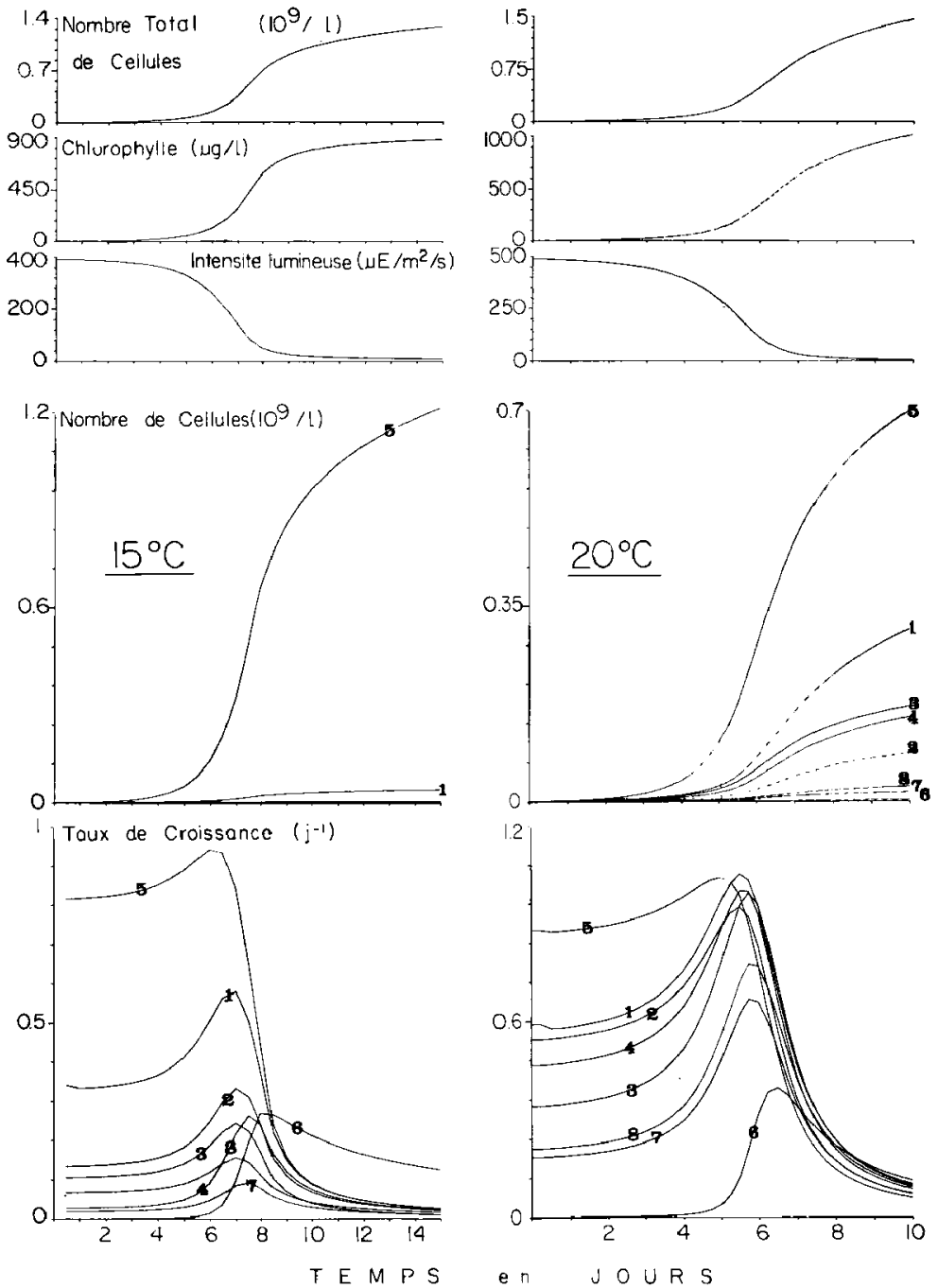

Fig. 6 a. Cultures plurispécifiques. Nutriments en excès.

Nombre initial de cellules/espèce $=10^{5} \cdot 1^{11}$.

1: Scenedesmus crassus 2: Coelastrum microsporum 3: Dictyosphaerium pulchellum 4 : Scenedesmus quadricauda 5 : Fragillaria bidens 6 : Anabaena cylindrica $7:$ Pediastrum boryanum 8: Monoraphidium minutum 

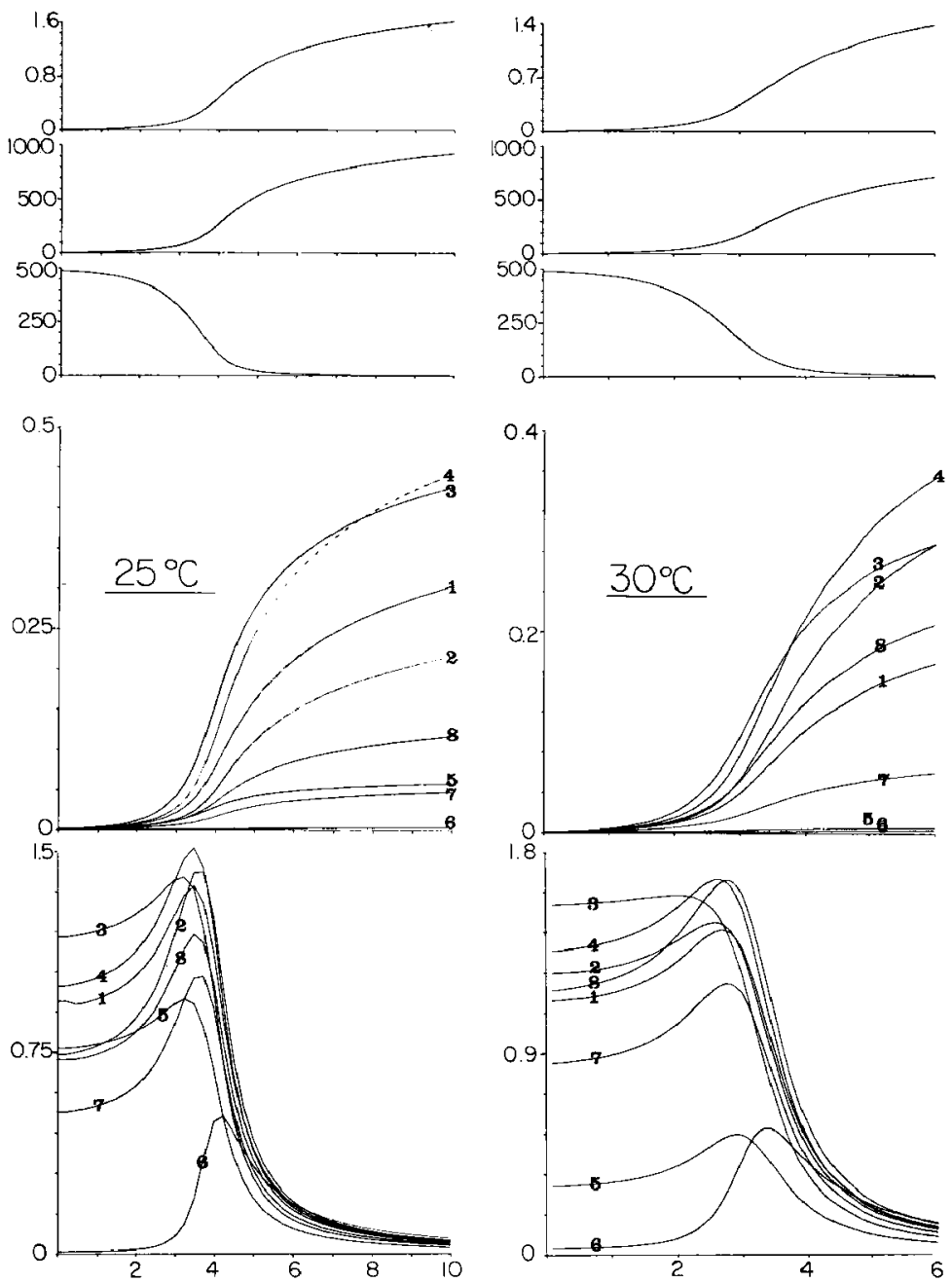

Fig. 6 b. Cultures plurispécifiques. Nutriments en excès.

Nombre initial de cellules/espèce $=10^{5}, 1^{11}$.

1: Scenedesmus crassus 2: Coelastrum microsporum 3 : Dictyosphaerium pulchellum 4 : Scenedesmus quadricauda 5 : Fragillaria bidens $6:$ Anabaena cylindrica 7 : Pediastrum boryanum 8 : Monoraphidium minutum 
de lá lumière) conduirait à une autre composition qualitative de la population.

\subsection{2. - Conditions limitantes en nutriments.}

Deux cas ont été similés sur une population plurispécifique carencée soit en azote (fig. 7), soit en phosphore (fig. 8) et perturbée par un ajout de l'élément absent.

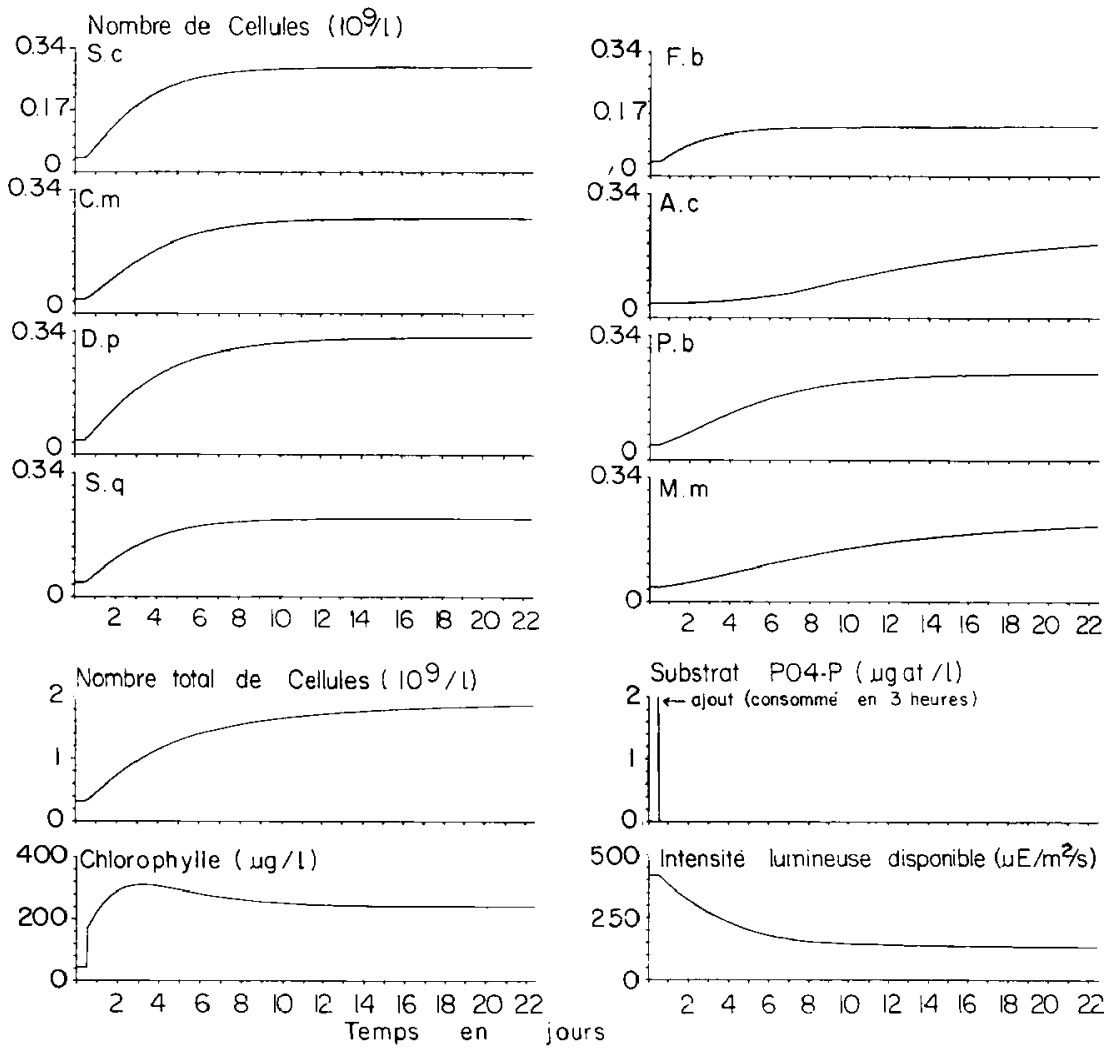

Fig. 7. Culture plurispécifique. Carence et ajout d'azote. Simulation.

S.c : Scenedesmus crassus C.m : Coelastrum microsporum D.p : Dictyosphaerium pulchellum S.q : Scenedesmus quadricauda F.b : Fragillaria bidens A.c : Anabaena cylindrica P.b : Pediastrum boryanum M.m : Monoraphidium minutum 

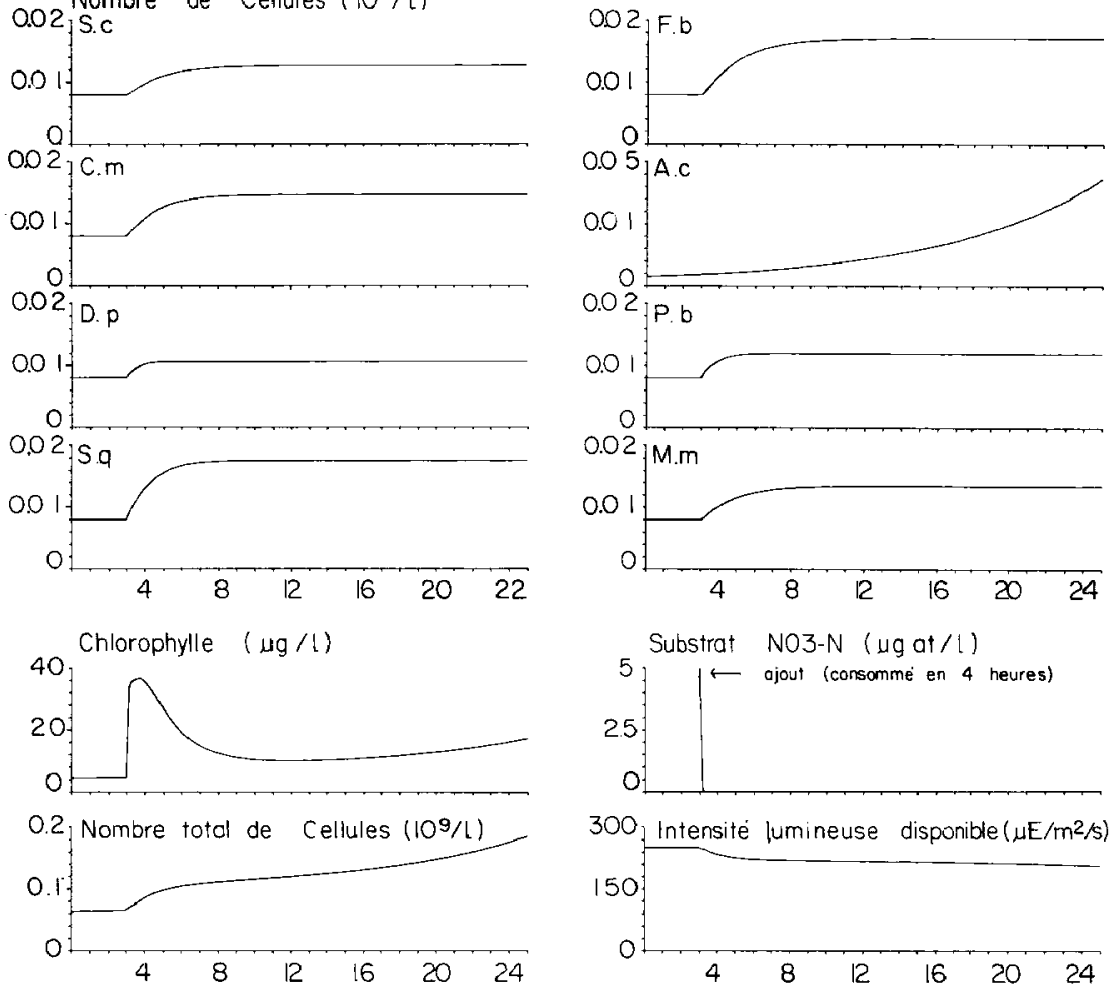

Temps en jours

Fig. 8. Culture plurispecifique. Carence et ajout de phosphore. Simulation.

S.c : Scenedesmus crassus C.m : Coelastrum microsporum D.p: Dictyosphaerium pulchellum $\mathbf{S . q}$ : Scenedesmus quadricauda F.b : Fragillaria bidens A.c : Artabaena cylindrica P.b: Pediastrum boryanum M.m : Monoraphidium minutum 
ture) : ce phénomène est très net dans le cas de l'ajout en phosphore, pour Anabaena, Pediastrum et Monoraphidium (fig. 8b).

\section{5. - Discussion}

La compétition entre espèces a souvent été abordée et étudiée à travers les effets d'auto et hétéro antagonisme (Tassigny et Lefèvre 1971, Keating 1978), la production d'inhibiteurs de croissance (Harris 1971), les effets algostatiques (Gravila 1972). Cependant, même si ces interactions ont un effet significatif, leurs formulations en termes quantitatifs dans un modèle dynamique restent encore à établir. Les études en ce domaine sont rare et revêtent plutôt un caractère qualitatif ou descriptif, comme par exemple :

- l'effet bactérien de Chlorella et Scenedesmus (Dor 1976)

- interaction entre Microcystis et Chlorella (Lam et Silvester 1979), ou eritre Scenedesmus et Oscillatoria (Mur et al. 1978). Ces derniers adoptent d'ailleurs une démarche voisine de celle présentée ici, en considérant la compétition par rapport à l'utilisation de la lumière.

Le modèle utilisé ici est axé principalement sur la dépendance de la croissance vis à vis de la lumière. de la température, de l'assimilation des nutriments et réserves intracellulaires : ces divers processus se traduisent par une compétition interspécifique.

Une approche similaire a été développée par Tilman et Kilham (1976), Jones et al. (1978) et Mickelson et al. (1979), qui comparent les résultats de cultures en batch ou semi-continues avec un modèle de compétition basé sur les nutriments.

Les modèles traitant de cultures monospécifiques sont plus nombreux, par exemple pour Scenedesmus et Chlorella (Azad et Borchardt 1970), Selenastrum (Toerien et Huang. 1973 a et b), Oscillatoria (Grenney et al. 1973). Cryptomonas (Cloern 1977), Oscillatoria (Ahlgren 1978), Skeletonema (Davis et al. 1978), Nostoc et Selenastrum (Brown et Harris 1978), Monochrysis (Burmaster 1979).

Certains aspects de la croissance en culture ou de l'assimilation des nutriments sont parfois pris en compte : Falkowsky (1977) utilise une constante d'affinité lumineuse pour le taux de consommation des nitrates. L'importance de la turbulence en culture
(Krueger et Eloff 1978), de la carence en carbone (Claesson et Forsberg 1978, Marvan et Pribil 1978) et les phénomènes d'ombre propre (Myers 1953, Marvan et Pribil 1978) sont rarement intégrés aux formulations.

Il est évident que des expressions telles la réduction du taux de croissance, ou la loi de MichaelisMenten pour l'assimilation, ne constituent que des approches imparfaites (Brown et al. 1978). Il apparaît également que les paramètres employés dans les formulations ne sont pas exempts d'incertitude :

- selon le système utilisé pour leur détermination, batch ou chémostat (Zevenboom et Mur 1979)

- selon le protocole adopté, Zevenboom et Mur (1978) font par exemple une distinction entre jeûne et carence.

- au niveau de la calibration (Lederman et al. 1976)

Pour certains processus, une tendance générale peut ètre dégagée : par exemple, le lien entre le taux d'assimilation et le rapport surface/volume d'une cellule, ou entre les possibilités de stockage et le biovolume. Il n'en est pas de même pour d'autre paramètres : Burmaster (1979) montre que la conception d'un taux de croissance instantané relié au quota est défectueuse : lors d'un ajout en nutriment, le modèle prédit une reprise de la croissance en avance sur les observations. Ce même type de remarque peut étre fait sur les résultats présentés ici, au sujet de la modélisation du quota en chlorophylle. La forme de stockage, inorganique ou inorganique (Goldman et Peavey 1979) et les cinétiques d'incorporation de ces pools à la matière organique vivante sont encore mal évaluées: Davis et al. (1978) utilisent une vitesse d'assimilation liée au substrat et une vitesse d'utilisation liée au stock interne de silice. Les modèles à plusieurs compartiments internes (Cloern 1976, Davis et al. 1978) sont certainement plus performants dans ce cas ; cependant, la mise en oeuvre de tels modèles suppose l'introduction de nouveaux paramètres difficiles à évaluer.

Le modèle présenté n'apporte en ces domaines que peu d'innovations, si ce n'est une inféodation de tous les paramètres à la température, et une expression de la teneur en chlorophylle, qui n'est plus considérée comme une constante mais qui devient une variable reliée à l'état nutritionnel. Tett et al. (1975) avaient également utilisé une formulation un peu analogue pour décrire le quotient chlorophylle/carbone. 
La bonne adéquation des résultats de la simulation avec les données expérimentales est enregistrée pour les phénomènes suivants :

- consommation rapide des nutriments

- consommation de luxe et stockage

- croissance différée par rapport à l'assimilation

- réduction de la croissance par l'ombre propre

- baisse de la teneur en chlorophylle dans le cas de limitation sévère en nutriment

- succession des espèces sans prise en compte des effets d'auto et hétéroantagonismes.

Yoder (1979) arrive à des résultats similaires, et montre en effet qu'un modèle prenant seulement en compte la lumière et la température, et basé sur des paramètres établis expérimentalement, est souvent suffisant pour expliquer les dynamiques observées dans ses expériences de cultures en dialyse.

Ces résultats montrent qu'un tel modèle basé sur les concepts actuels et grâce à une formulation synthétique- permet de rendre compte pour une grande part des cinétiques d'assimilation et de croissance de populations d'algues mono ou plurispécifiques. Ceci est en grande partie lié à la mise en oeuvre d'un modèle basé sur des paramètres interdépendants qui apportent fiabilité et robustesse.

Des progrés restent cependant à faire, en particulier pour les modélisations à court terme (que)ques heures à un jour) dans le cas de populations carencées et perturbées par un ajout de nutriment.

Plus généralement, l'amélioration de l'aptitude de ces modèles à simuler la dynamique de peuplements phy toplanctoniques dans des conditions très diverses suppose :

- la recherche d'une formulation, simple mais généralisable, des processus écophysiologiques qui contrôlent la croissance et le déclin des populations. Il convient en particulier de baser cette formulation sur des paramètres accessibles aux mesures de terrain, en recherchant les corrélations entre les paramètres et leur signification.

- en conséquence, le développement d'études expérimentales destinées à mieux comprendre les variations de la composition biochimique des algues et leurs effets sur les fonctions métaboliques.

Il est ainsi facile de démontrer que les modèles de simulation - en dehors de leur utilisation en tant qu'outils de prédiction et de synthèse des connaissances - peuvent jouer un róle interactif en indiquant au chercheur les phénomènes à préciser et à mieux définir.

\section{Travaux cités}

Ahlgren (G.). 1978. - Growth of Oscillatoria agardhit in chemostat culture. 2. Dependance of growth constants on temperature. Mitt. Intemat. Verein. Limnol, 21 : 88.102.

Azad (H.S.) \& Borchardt (J.A.). 1970. - Variations in phosphorus uptake by algae. Environ. Sci. Technol, 4 (9) : $737-743$.

Belyanyn (V.N.) Kovrov (B.G.). 1968. - Mathematical model of biosynthesis in a light-limited microalgal culture. Dokl. Akad. Nauk. SSSR., 179:1463-1466.

Brown (E.J.), Harris (R.F.) \& Koonce (J.F.). 1978. - Kinetics of phosphate uptake by aquatic microorganisms : deviations from a simple Michaelis-Menten equations. Limnol. Oceanogr., 23 (1): $26-34$.

Brown (E.J.) \& Harris (R.F.). 1978. - Kinetics of algal transient phosphate uptake and cell quota concept. Limnol. Oceanogr., $23(1): 35 \cdot 40$.

Brunel (L.), Dauta (A.) \& Guerri (M.M.). 1982. - Croissance algale : Validation d'un modèle à stock à l'aide de données expérimentales. Annis Limnol. $18(2): 91-100$.

Burmaster (D.E.). 1979. - The unsteady continuous culture of phosphate-limited Monochrysis lutherii Droop. experimental and theoritical analysis. J. Exp. Mar, Ecol, 39 (2): 167-186.

Caperon (J.). 1967. - Population growth in microorganisms limited by food supply. Ecology, $48 ; 715-722$.

Chu (S.P.). 1942. - The influence of the mineral composition of the medium on the growth plarkton algae. Part. I. Methods and culture media. J. Ecol., $30: 284-325$.

Chu (S.P.). 1943. - The influence of the mineral composition of the medium on the growth of plankton algae. Part. II. The influence of the concentration of inorganic nitrogen and phosphate phosphorus. J. Ecol., 31 (2) : 109-148.

Claesson (A.) \& Forsberg (A.). 1978. - Algal assay procedure with one or five species. Minitest. Mitt. Intemat. Verein. Limnol. $21: 21-30$.

Cloem (J.E.). 1977. - Effects of light intensity and temperature on Cryptomonas ovata (Cryptophyceae) growth and nutrient uptake rates. J. Phycol., 13 (4) : 389-395.

Cobb (H.D.) \& Myers (J.). 1965. - Comparative studies of nitrogen fixation and photosynthesis in Anabaena cylindrica. Am. J. Bot., $51: 733.762$.

Coombs (J.). Darley (X.M.), Holm-Hansen (O.) \& Volcani (B.E.). 1976. - Chemical composition of Navicula pelliculosa during silicon-starvation synchrony. Pl. Physiol., 42 : $1601-1606$.

Dauta (A.) 1983a. - Conditions de développement du phytoplancton. Etude comparative du cumportement de huit especes en culture. I. Détermination des paramètres de croissance en fonction de la lumière et de la température. Annls Limnol. $18(3): 217-262$.

Dauta (A.) 1983b. - Conditions de développement du phytoplancton. Etude comparative du comportement de huit especes en culture. II. Role des nutriments : Assimilation et stockage intracellulaire. AnnIs Limnol., 18 (3) : 263.292.

Davis (C.O.), Breitner (N.F.) \& Harrison (P.J.). 1978. - Continuous culture of marine diatoms under silicon limitation. 3. A model of Si-limited diatom growth. Limnol. Oceanogr., 23 (1): 41-52.

Dor (I.). 1976. - Why Chlorella and Scenedesmus are the dominant algae in the wastewaters over the world : a hypothesis. In Proceedings of the 7th Scientific conference of the Israel Ecological Society. Tel Aviv. Publ by IES, Tel Aviv., p. : 13-25.

Droop (M.R.). 1968. - Vitamin B12 and marine ecology. 4. The kinetics of uptake. growth and inhibition in Monochrysis Iutherii. J. Mar. Biol. Assoc. U.K., $48: 689.733$.

Droop (M.R.). 1973. - Some toughts on nutrient limitation in algae. J. Phycol., $9(3): 264-273$.

Dugdale (R.C). 1967. - Nutrient limitation in the sea : dynamics. identification and significance. Limnol. Oceanogr., 12 : 685-695. 
Eppley (R.W.) ; Rogers (J.N.) \& Mccarthy (J.J.). 1969. - Half saturation constants for uptake of nitrate and ammonium by marine phytoplankton. Limnol. Oceanogr., 14:912-920.

Falkowski (P.G.). 1977. - A theoritical description of nitrate uptake kinetics in marine phyloplankton based on biosubstrate kinetics. J. Theor. Biol., 64. : 375-379.

Goldman (J.C.) \& Peavey (D.G.). 1979. - Steady-state growth and chemical composition of the marine Chlorophyte Dumaliella tertiolecta in nitrogen-limited continuous cultures. Appl. Environ. Microbiol. 38 (5) : 894-901.

Gramms (L.C.) \& Boyle (W.C.). 1972. - Reflectance and transmit tance characteristics of selected green and blue-green algae. Water Res., 6(12). : 1433-1444.

Gravila (L.). 1972. - Aspecte competitive la algae. Bull. Cercet. Piscic., 31(1-2): $31-38$

Grenney (W.S.), Bella (D.A.) \& Curl (H.C.). 1973. - A theoritical approach to interspecific competition in phytoplankton communities, Am. Nat., 107 (955) : 485-425.

Harris (D.0.). 1971. - Growth inhibitors produced by the green algae (Volvocaceae). Arch. Mikrobiol., 76(1) : 47-50.

Healey (F.P.) \& Hendzel (L.L.). 1975, - Effects of phosphorus deficiency on two algae growing in chemostats. $J$. Phycol., $11(3): 303-309$

Holmes (R.W.). 1966. - Light microscope observations on cytological manisfestation of nitrate, phosphate and silicate deficiency in four marine centric Diatoms. $J$. Phycol., $2: 136-140$.

Horne (A.J.), Sandusky (J.C.) \& Carmiggelt (G.J.W.). 1979. Nitrogen fixation in Clear Lake, California. 3. Repetitive synoptic sampling of the Aphanizomenon blooms. Limnol. Oceanogr., 24 (2) : 316-328.

Jones (K.J.). Tett (P.), Wallis (A.C.) \& Wood (W.C.B.). 1978. Investigation of a nutrient-growth model using a continuous culture of natural phytoplankton. I. Mar. Biol. Assoc. U.K., $58(4)$ : $923-921$

Keating (K.I.). 1978. - Blue green algae inhibition of diatom growth : transition from mesotrophic to eutrophic community structure. Science, 199:971-973.

Krueger (G.H.J.) \& Eloff (J.N.). 1978. - The effect of agitation and turbulence of the growth medium on the growth and viability of Microcystis. J. Limnol. Soc. Sotuth Afr. 4 (1):69-74.

Lam (C.W.Y.) \& Silvester (W.B.). 1979. - Growth interactions among blue-green (Anabaena Oscillarioides, Microcystis aeruginosa) and green (Chlorella sp.) algae. Hydrobiologia., 63 (2) : 135.143.

Lederman (T.C.), Homberger (G.M.) \& Kelly (M.G.). 1976. - The calibration of a phytoplankton growth model using batch culture data. Water Air Soil Pollut., 5 (4) : 431442.

Lehman (J.T.), Botkin (D.B.) \& Likens (G.E.) 1975. - The asump. tions and rationales of a computer model of phytoplankton population dynamics. Limnol. Oceanogr., 20:343-364.

Livansky (K.). 1979. - Productivity of algal suspensions in cultivation systems with different geometry. Arch. Hydrobiol. (Suppl.,), 56 (3) : 339-346.
Marvan (P.) \& Pribil (S.). 1979. - Factors limiting the growth of algal cultures. In Algal and Monitoring Eutrophication, Marvan P., Pribil S., Lhotsky O. (Eds.). E. Schweizerbart, Stuttgart, $p: 47.56$.

Mickelson (M.J.), Maske (H.) \& Dugdale (R.C.). 1979. - Nutrientdetermined dominance in multispecies chemostat cultures of Diatoms. Limnol. Oceanogr., 24 (2) : 298-315.

Mur (L.R.), Gons (H.J.) \& Van Liere (L.). 1978. - Competition of the green alga Scenedesmus and the blue-green alga Oscillatoria. Mitt. Internat. Verein. Limnol. Oceanogr.. $21: 473.479$.

Myers (1.7. 1953. - Growth characteristics of algae in relation to the problem of mass culture. In Algal Culture from Laboratory to Pitot Plant. Burlew J.S., (ed.), Carnegie Institution of Washington Publication, 600 : 37-54.

Nyholm (N.). 1979. - A mathematical model for growth of phytoplankton. Mitt. Internat. Verein. Limnol., 21: 193-206.

Ogawa (R.E.) \& Carr (J.F.). 1969. - The influence of nitrogen on heterocyst production in blue-green algae. Limnol. Oceanogr.. $14: 342.351$.

Rodhe (W.). 1948. - Environmental requirements of freshwater phytoplankton algae. Experimental studies in the ecology of phytoplankton. Symb. Bor. Upsat., 10 (1): 1-149.

Steele (J.H.). 1962. - Environment cont rol of photosynthesis in the sea. Limmol. Oceanogr., 7: 137-150.

Tassigny (M.) \& Lefevre (M.). 1971. - Auto., hétéroantagonisme et autres conséquences des excrétions d'algues d'eau douce ou thermale. Verh. Intemat. Verein. Limnol., 19 (1): 26-38.

Tett (P.), Cottrel (J.C.), Trew (D.O.) \& Wood (B.J.B.). 1975. Phosphorus quota and the chlorophyll : carbon ratio in marine phytoplancton. Limnol. Oceanogr., 20 (4) : 587-603.

Tilman (D.) \& Kilham (S.S.). 1976. - Phosphate and silicate growth and uptake kinetics if the Diatoms Asterionella formose and Cyclotella meneghiniana in batch and semi-cont inuous culture. J. Phycol., $12: 375.383$.

Toerien (D.F.) \& Huang (C.H.) 1973. - Algal growth prediction using growth kinetics constants. Wat. Res., 7: 1673-1681.

Werner (D.). 1977. - Silicate metabolism. In : The Biology of Diatoms.., Werner D., (ed.), Botanical Monographs., 13 $111+149$.

Yoder (J.A.) 1979. - A comparison between the cell division rate of natural populations of the marine Diatom Skeletonema costatum (Greville) Cleve grown in dialysis culture and that predicted from a mathematical model. Limnol. Oceanogr., 24 (1) : 97-106.

Zevenboom (W.) \& Mur (L.R.). 1978. - N-uptake and pigmentation of N-limited chemostat cultures and natural populations of Oscillatoria agardhii. Mitt. Intemat. Verein. Limmol, 21 : 261-274.

Zevenboom (W.) \& Mur (L.R.). 1979. - Influence of grow th rate on short term and steady-state nitrate uptake by nitratelimited Oscillatoria agardhit. Fems Microbiol. Lett., 6 (4): 208-212. 